\title{
MODE OF ACTION OF GUANETHIDINE ON ADRENERGIC NEURONS AND ITS DEPENDENCE ON SODIUM
}

\author{
Takao KUBO* and Yoshimi MISU** \\ Department of Pharmacology, Faculty of Pharmacentical Sciences, \\ Kyoto University, Sakyo-ku, Kyoto
}

Accepted September 25, 1973

\begin{abstract}
Interactions between action of guanethidine $\left(4 \times 10^{-6}\right.$ to $\left.4 \times 10^{-5}\right)$ and $\mathrm{Ca}$, $\mathrm{Na}$ or $\mathrm{K}$ were investigated in isolated hearts of rabbits. Positive chronotropic responses to electrical sympathetic stimulation and noradrenaline (NA) released by nicotine or high $\mathrm{KCl}$ were measured. Inhibitory drug action on responses was irreversible and reduced by raising $\mathrm{Ca}$ to $12.5 \mathrm{mM}$ together with drug addition. The action was not reduced after the drug had been washed out and the Ca level was raised to $50 \mathrm{mM}$, after which it returned to normal. It was accelerated by lowering $\mathrm{Na}$ to $86 \mathrm{mM}$ simultaneously, but not delayed by raising $\mathrm{Na}$ to $286 \mathrm{mM}$. After the drug was washed out with normal solution, NA released by 100 and $300 \mathrm{mM} \mathrm{KCl}$ was reduced to almost the same extent. When stinulation was done during washing with low Na solution, inhibitory drug action on responses and NA relcascd by nicotine was reduced. It was not reduced, however, when nicotine was applied in normal $\mathrm{Na}$ solution after washing with low $\mathrm{Na}$ solution. During washing with high $\mathrm{Na}$ solution, it was accentuated, despite no modification by washing with isoosmotic sucrose or Li solution. Low or high $\mathrm{Na}$ solution alone did not modify the parameters. During washing with low $\mathrm{K}$ solution $(3.5 \mathrm{mM})$, it was not modified. The hypothesis is presented that guanethidine increases permeability of norve ending membrane to $\mathrm{Na}$, thereby leading to adrenergic neuron blockade.
\end{abstract}

Since the early work of Maxwell et al. (1), the antihypertensive action of guanethidine has been attributed to its adrenergic ncuron blockade. Kirpekar et al. (2) demonstrated that in the guanethidine-perfused spleen of cats, high calcium restored the noradrenaline released in response to splenic nerve stimulation. Boullin (3) reported that calcium increased the release of radioactive guanethidine in the heart of rats. On the other hand, it was reported by Wilson (4) and Chang et al. (5) that the antagonism between calcium and guanethidine on contractile responses to sympathetic nerve stimulation was physiological in nature, in the ileum and vas deferens of rats. Boura and Green (6) suggested the possibility that a depressant action of adrenergic neuron blockers in the nerve terminals was analogous to the impairment of conduction in the nerve trunks caused by local anesthetics.

Whether or not an essential interaction exists between the action of guanethidine and calcium, sodium or potassium was investigated herein utilizing the heart of rabbits. The hypothesis has been presented that guanethidine increases the permeability of the

* Present address; Fujisawa Laboratory, Fujisawa Pharmaceutical Co., Ltd., Higashiyodogawaku, Osaka, Japan.

** To whom requests for reprints should be addressed. 
membrane of nerve endings to sodium ions, thereby leading to adrenergic neuron blockade. Some of these findings were presented at the 38th (7) and 39th (8) Regional Meeting of the Japanese Pharmacological Society, Kinki area.

\section{MATERIALS AND METHODS}

About 120 rabbit hearts of both sexes weighing 1.7 to $2.4 \mathrm{~kg}$ were used. The bilaterat sympathetic trunks (mainly the stellate ganglia) were isolated carefully from the surrounding connective tissues and ligated in the same experimental conditions as described previously by Misu et al. (9). In some experiments, the sympathetic nerve atrial preparations were prepared as described by Misu and Kirpekar (10). The nerves were held by fluid electrodes similar to those described by Garry and Gillespie (11), i.e. a pair of loops of silver wire encased in plastic cell-walls. The preparation was suspended in the muscle chamber containing Krebs bicarbonate solution, bubbled with $95 \% \mathrm{O}_{2}$ and $5 \% \mathrm{CO}_{2}$ maintained at $30^{\circ} \mathrm{C}$, having the following composition (expressed in $\mathrm{mM}$ ): $\mathrm{NaCl} 118.4 ; \mathrm{KCl} 4.7 ; \mathrm{CaCl}_{2}$ 2.5; $\mathrm{MgCl}_{2} 7 \mathrm{H}_{2} \mathrm{O} 1.18 ; \mathrm{NaHCO}_{3} 25 ; \mathrm{KH}_{2} \mathrm{PO}_{4} 1.2$; and glueose 11.1. The final $\mathrm{pH}$ was approx. 7.5. Most experiments, however, were done in the Langendorff's preparation, which was prepared as described previously (9). Perfusion was done mainly at a constant pressure of $60 \mathrm{~cm} \mathrm{H}_{2} \mathrm{O}$ and $30^{\circ} \mathrm{C}$. The fluid electrodes were used with a modification for the stimulation of the stellate ganglia in this preparation. Both cells of the electrodes were partitioned with a thin rubber diaphragm. One half of the cell was filled with Krebs solution bubbled with the mixed gas and sealed off with a rubber membrane and a plastic disk with an eyelet of approx. $2 \mathrm{~mm}$ diameter in the center. The ligature attached to the stcllate ganglia was threaded, by means of a needle, through the eyelet and the rubber diaphragms. The threaded nerves were pulled into the electrodes. The other half cell was then filled with Krebs solution and sealed off with a disk. The thread attached to the stellate ganglia was held between the disk and the casing of the electrodes. The preparation was then suspended from the perfusion apparatus (Natsume KN-206). Spontaneous contraction was recorded on a smoked paper with a semi-isotonic lever. In both preparations of atria and perfused hearts, the bilateral stellate ganglia were stimulated with an electronic stimulator MSE-3 (Nihon Kohden), for $30 \mathrm{sec}$ with supramaximal rectangular pulses of $3.5 \mathrm{msec}$ duration at a frequency of $30 \mathrm{cps}$. Heart beats/min were counted.

In some perfused hearts, the sympathetic nerve endings were stimulated by perfusion with nicotine solution $\left(4 \times 10^{-5} \mathrm{M}\right)$ for 2 min or with $\mathrm{KCl}$ solution $(100$ and $300 \mathrm{mM}$ ) for 4 min in the same manner as described previously (9). In the latter case, perfusion was performed at a constant volume $(10 \mathrm{ml} / \mathrm{min})$ by means of a perfusion pump (Iwashiya). The output of noradrenaline released into the venous effluent was measured fluorometrically by the method of Anton and Sayre (12) using a spectrofluorophotometer GF-16 (Shimazu). Recovery was approx. 60\%.

A modified Krebs solution containing 215 and $286 \mathrm{mM}$ sodium, $143 \mathrm{mM}$ lithium, $3.5,23.6,100$ and $300 \mathrm{mM}$ potassium or $12.5 \mathrm{mM}$ calcium was prepared by an addition 
or removal of each hydrochlorate without altering the relation of the other electrolytes and compensating changes in tonicity. In order to make a $50 \mathrm{mM}$ calcium Krebs solution, $\mathrm{NaHCO}_{3}$ was eliminated and $\mathrm{pH}$ was adjusted to 7.0 with $1 \mathrm{~N} \mathrm{KOH}$. A $86 \mathrm{mM}$ sodium solution was prepared by isoosmotic replacement of sodium chloride $(57 \mathrm{mM})$ by sucrose or glucose.

Drugs used were guanethidine sulphate (Ciba-Geigy), nicotine bitartrate (Tokyo Kasei), cocaine hydrochloride (Takeda) and procaine hydrochloride (Takeda), which are expressed as molar concentrations.

Peak increases of heart rate in responses to electrical stimulation of the sympathetic nerve and the output of noradrenaline released during the perfusion with nicotine or $\mathrm{KCl}$ solution before administration of guanethidine are designated as $100 \%$ control responses. Student's t-test was used to evaluate data.

\section{RF.SULTS}

Effects of high calcim on granethidine-induced decrease in positive chronotropic responses to sympathetic nerve stimulation in the isolated atrium of rabbits

Electrical stimulation of sympathetic nerve markedly increased contractile rate and force of the spontaneously beating atria. Percent increases in chronotropic responses were $55.2 \pm 8.3(n-13)$. Guancthidine was added to the organ bath. A concentration of $4 \times 10^{-5} \mathrm{M}$ of the drug produced a slight increase in atrial contractile rate and force. The percent increase in atrial rate was $11.3+1.0(n-6) 30$ min after the addition. As demonstrated in Table 1 , section $\mathrm{B}$, guanethidine reduced atrial positive chronotropic responses to the 2nd nerve stimulation $30 \mathrm{~min}$ after the 1 st stimulation $108.2 \pm 1.3 \%$ of control responses. The drug was washed away with normal fresh solution. Responses, which had been reduced by the pretreatment with guanethidine, were not reversible but reduced progressively 30 min after the washing (section $C$ ). In section $\mathbf{D}$, the concentration of calcium was raised to totally $12.5 \mathrm{mM}$ together with the addition of guanethidine.

TABI.r. 1. Effects of high calcium on guanethidine-induced decrease in positive chronotropic responses to electrical stimulation of sympathetic nerve in the isolated atria of rabbit.

\begin{tabular}{|c|c|c|c|c|}
\hline Procedures & $\begin{array}{l}\text { No. of } \\
\text { Exp. }\end{array}$ & $\begin{array}{l}\text { Guanethidine } \\
\text { (M) }\end{array}$ & $\begin{array}{l}\text { Calcium } \\
(\mathrm{mM})\end{array}$ & $\begin{array}{l}\text { Positive chrono- } \\
\text { tropic responses } \\
\text { (mean } \pm \text { s.e.) } \\
\qquad(\%)\end{array}$ \\
\hline A. Initial nerve stimulation & 13 & 0 & 2.5 & 100 \\
\hline B. In the presence of guanethidinc & 6 & $4 \times 10^{-5}$ & 2.5 & $8.2 \pm 1.3$ \\
\hline C. After washing out guanethidine & 3 & $4 \times 10^{-5}$, then 0 & 2.5 & $2.2 \pm 1.8$ \\
\hline $\begin{array}{l}\text { D. In the presence of guanethidine } \\
\text { and high calcium }\end{array}$ & 4 & $4 \times 10^{-5}$ & 12.5 & $34.6 \pm 12.3$ \\
\hline E. After washing out high calcium & 3 & 0 & 50.0 , then 2.5 & $95.3 \pm 9.2$ \\
\hline $\begin{array}{l}\text { F. After incubation first with } \\
\text { guanethidine, then with high } \\
\text { calcium }\end{array}$ & 3 & $4 \times 10^{-5}$, then 0 & $\begin{array}{c}50.0 \text { (drug free), } \\
\text { then } 2.5\end{array}$ & 0 \\
\hline
\end{tabular}

Stimulation was repeated at intervals of 30 to $60 \mathrm{~min}$ 
The percent increase in atrial rate was $27.9 \div 8.330 \mathrm{~min}$ after the addition. Responses to the 2nd nerve stimulation were less inhibited as compared with those after addition of the drug alone. In section $\mathrm{E}$, preparations were incubated with a high calcium solution (totally $50 \mathrm{mM}$ ). The high calcium markedly potentiated the atrial contractile force but reduced the rate, sometimes causing a standstill of spontaneous rhythm 10 to $15 \mathrm{~min}$ after incubation. The atria, however, were continuously incubated with the high calcium for $30 \mathrm{~min}$, and then were washed with normal calcium solution $(2.5 \mathrm{mM})$. The atrial rate, which had been reduced or abolished by the high calcium, was restored 30 min after the washing and the nerve stimulation produced responses as observed in the control. In section $F$, the atria were incubated with guanethidine for $30 \mathrm{~min}$, then with the drug-free high calcium solution $(50 \mathrm{mM})$ for $30 \mathrm{~min}$, after which washing with normal calcium solution was carried out. When the $3 \mathrm{rd}$ stimulation was carried out in the presence of normal solution $30 \mathrm{~min}$ after the washing, responses were nil.

Effect of low or high sodium on guanethidine-induced decrease in positive chronotropic responses to sympatheric nerve stimulation in the isolated heart of rabbits

In untreated perfused hearts, a percent increase in chronotopic responses to the initial nerve stimulation was $42.7 \pm 3.4(n=3)$. As demonstrated in Fig. 1-A, the nerve stimulation produced the same responses as the initial responses at least for $1 \mathrm{hr}$ after perfusion. When hearts were perfused with a solution containing $286 \mathrm{mM}$ sodium, contractile force and positive contractile responses began to decrease markedly 6 to $8 \mathrm{~min}$ after the perfusion. Contraction force increased slightly after perfusion with a solution containing $86 \mathrm{mM}$ sodium. Heart rate and positive chronotropic responses, however, did not show marked modifications until at least 20 min after the perfusion with 2 solutions containing modified concentrations of sodium. Thereafter, the responses gradually decreased.

As demonstrated by the solid line in Fig. 1-B, when hearts were perfused with normal sodium solution containing guanethidine $\left(4 \times 10^{-6}\right)$, responses to nerve stimulation decreased gradually. Positive chronotropic responses decreased to $28.7+10.6 \%$ of control 30 min after the perfusion $(n=6)$. When hearts were washed with fresh solution 30 min after the perfusion, responses were not reversible but reduced progressively. Responses $30 \mathrm{~min}$ after washing were $13.0 .15 .2 \%$ of control $(\mathbf{n}=3)$. As shown by the bottom line, the inhibitory action of guanethidine was accelerated by perfusion with the low sodium solution $(86 \mathrm{mM})$. The difference was significant $(\mathrm{P}<0.05)$ at 10,15 and 20 min after the perfusion from the action of the drug perfused with normal sodium solution (top line), respectively. The inhibitory action was not delayed or lessened but rather was slightly accelerated by the high sodium solution (middle line).

Perfusion with cocaine or procaine was attempted for comparison with the action of guanethidine. Concentrations of these local anesthetics required to produce similar inhibition of responses to that obtained with guanethidine $4 \times 10^{-6}$ were found to be $10^{-4}$ or more, which per se markedly depressed contractile rate and force. Responses, depressed by the local anesthetics, were restored almost completely 20 min after washing with fresh solution. 


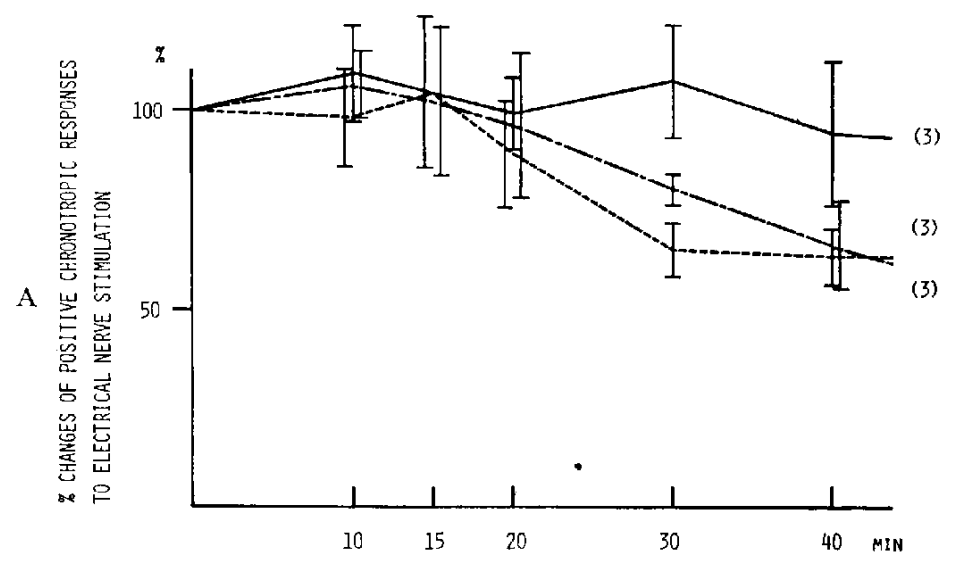

TIME AFTER CHANGING SOLUTIONS

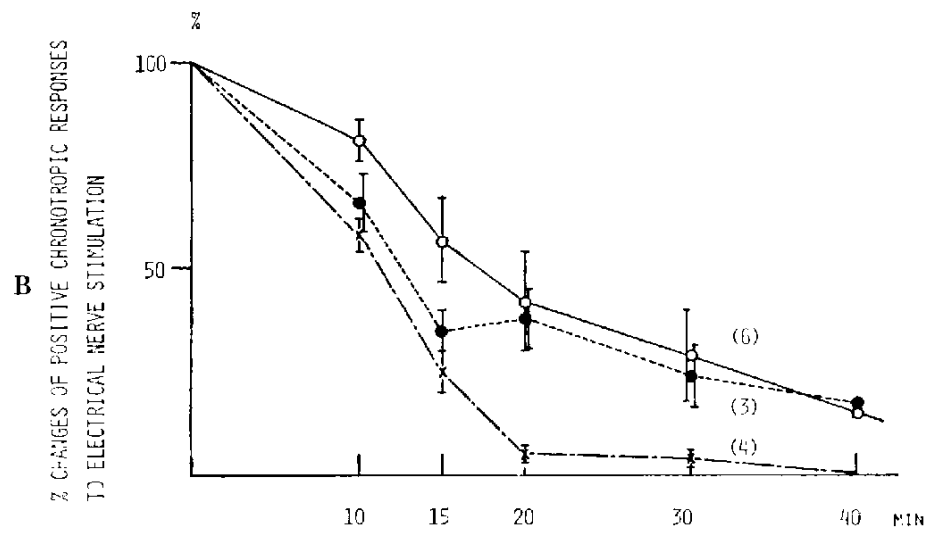

TIME AFTER PERFUSION OF GLANETHIJIIF: $\left(4 \times 10^{-6}\right.$, y)

FIG. 1. Effect of low or high sodium on guanethidine-induced decrease in positive chronotropic responses to electrical stimulation of sympathetic nerve in the perfused heart of rabbit.

Stimulation repeated each time is shown in abscissa. Responses to the 1st stimulation are taken as 100\% (Figs. 1 and 3-A). After the 1st stimulation, hearts were perfused with solution containing normal (143 mM, - -286 $(---)$ or $86 \mathrm{mM}(-\ldots)$ sodium alone (A) and guanethidine (B). Parentheses indicate number of experiments and vertical bars standard errors, these being common to all Figs.

Effect of guanethidine on output of noradrenaline released by high concentrations of $\mathrm{KCl}$ in the isolated heart of rabbits

When perfused with 100 or $300 \mathrm{mM} \mathrm{KCl}$ solution, hearts did not deteriorate in the hypertonic condition, but occasionally swelling was observed due to coronary constriction. Results obtained in such preparations were omitted. In untreated hearts, the 1st perfusion with 100 and $300 \mathrm{mM} \mathrm{KCl}$ solution released 28.2-259.8 $\mathrm{ng}$ (an average of 110.4 $\mathrm{ng}, \mathrm{n}=3$ ) and $67.8-672.0 \mathrm{ng}$ of noradrenaline (an average of $298.8 \mathrm{ng}, \mathrm{n}=3$ ), respectively. 


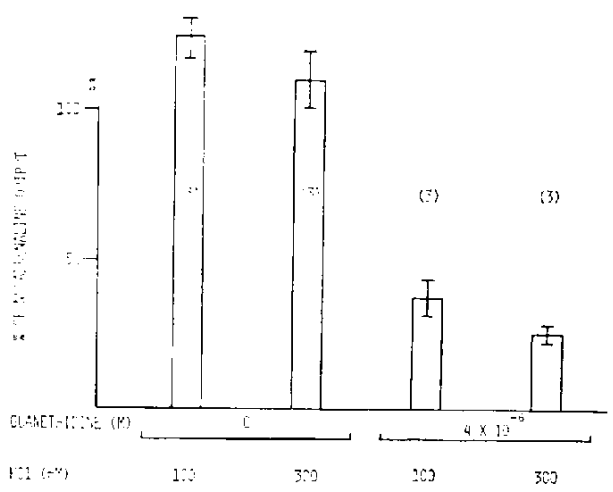

FIG. 2. Effect of guanethidine on output of noradrenaline released by high concentrations of $\mathrm{KCl}$ in the perfused heart of rabbit.

Perfusion with $\mathrm{KCl}$ solution was done twice at $30 \mathrm{~min}$ intervals. The output of noradrenaline released with the 1 st perfusion is taken as $100 \%$, this being common to Fig. 3-B.

In the two columns on the right, the 2 nd $\mathrm{KCl}$ solution was applied in fresh solution after perfusion with guanethidine solution for $20 \mathrm{~min}$.

As demonstrated in the left half of Fig. 2, the 2nd perfusion with 100 and $300 \mathrm{mM} \mathrm{KCl}$ solution released $124.1 \pm 7.2$ and $109.9 \pm 9.0 \%$ of the noradrenaline with the 1 st perfusion, respectively. Perfusion with a solution containing equiosmotic amount of glucose to the $300 \mathrm{mM} \mathrm{KCl}$ solution did not release a measurable amount of noradrenaline $(\mathrm{n}=$ 2). The limit of the sensitivity of the present chemical assay of noradrenaline was approx. $10 \mathrm{ng}$. In the right half, when the 2 nd 100 and $300 \mathrm{mM} \mathrm{KCl}$ solution was perfused in fresh solution $5 \mathrm{~min}$ after washing out the guanethidine solution $\left(4 \times 10^{-8}\right)$, the output of noradrenaline was markedly reduced to $37.6 \pm 6.1$ and $25.7 \pm 2.9 \%$ of that released with the 1st perfusion, respectively.

Effect of washing with low or high sodium solution on guanethidine-induced decrease in positive chronotropic responses to sympathetic nerve stimulation in the isolated heart of rabbits

In Fig. 3-A, when the 2 nd nerve stimulation was carried out $10 \mathrm{~min}$ after washing out the guanethidine solution $\left(4 \times 10^{-6}\right)$ with normal sodium solution, responses were $45.0 \pm$ $4.0 \%$ of those obtained with the 1 st stimulation (lined column). This value was regarded as control of the drug action, which remained after the drug had been washed out. The action of guanethidine was reduced significantly $(\mathrm{P}<0.05)$ by washing with the solution containing $86 \mathrm{mM}$ sodium, being made equiosmotic by addition of sucrose. On the other hand, it was accentuated significantly $(\mathrm{P}<0.01)$ by washing with a solution containing $286 \mathrm{mM}$ sodium, but not modified by washing with a solution containing an isoosmotic amount of sucrose $(251 \mathrm{mM})$ or lithium chloride $(143 \mathrm{mM})$.

Effect of washing with low or high sodium solution on guanethidine-induced decrease in output of noradrenaline released by nicotine in the isolated heart of rabbits

In untreated hearts, the 1 st perfusion with nicotine solution $\left(4 \times 10^{-5}\right)$ released $185.5-$ $2131.5 \mathrm{ng}$ of noradrenaline (an average of $1059.6 \mathrm{ng}, \mathrm{n}=7$ ). As demonstrated in the ex- 

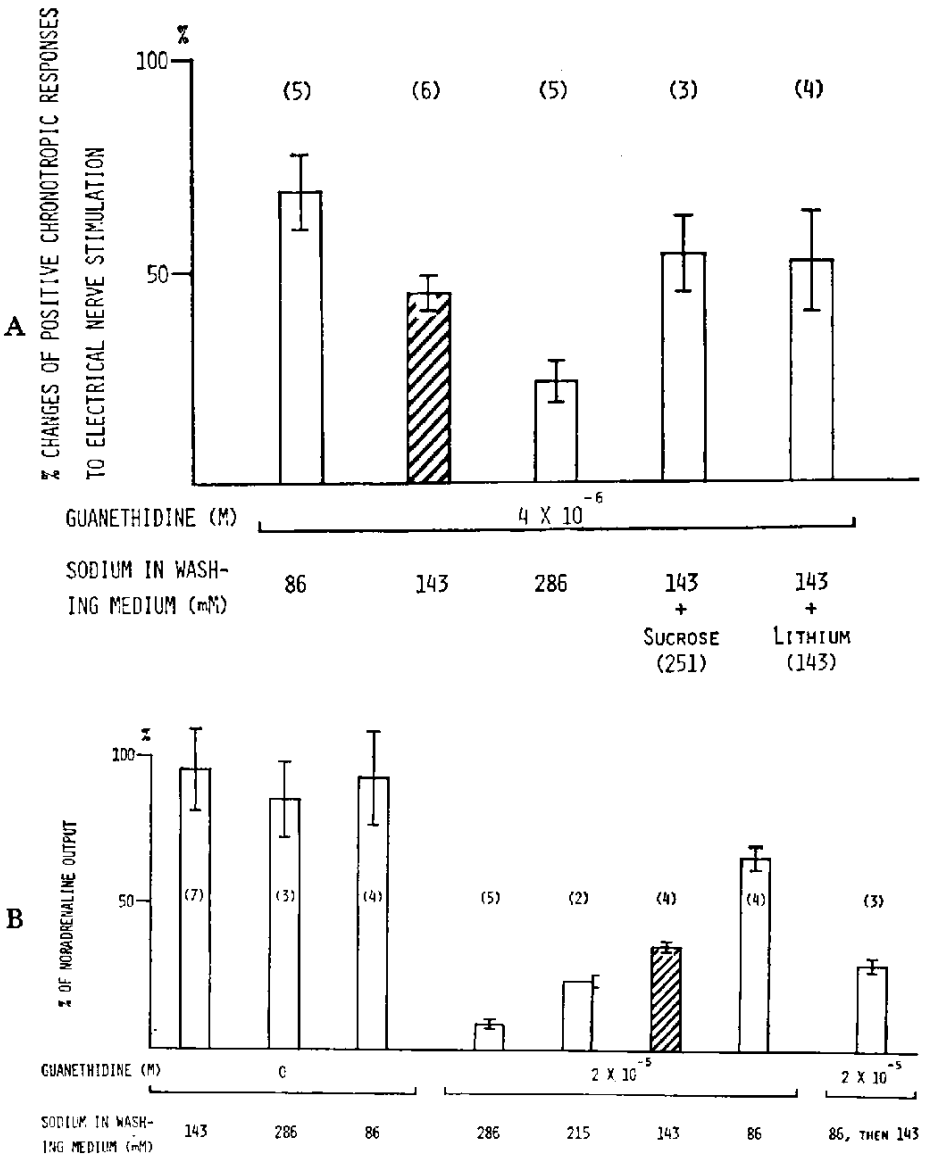

FIG. 3. Effects of washing with low or high sodium solution on guanethidine-induced decrease in positive chronotropic responses to electrical stimulation of sympathetic nerve and in the output of noradrenaline released by nicotine in the perfused heart of rabbit.

Electrical stimulation (A) or perfusion with nicotine solution (B) was carried out twice, usually at 30 min interval. After the 1st procedure, hearts were perfused with guanethidine solution ( $A$, middle and right columns of $B$ ) or normal solution (left columns of B) for $20 \mathrm{~min}$, and then washed with washing medium described in abscissa. The 2nd procedure was done during perfusion with washing medium. Horizontal levels of the bracket bar represent the actual $\%$ of noradrenaline.

treme left column of Fig. 3-B, the 2nd perfusion with nicotine released $95.3-14.1 \%$ of the noradrenaline obtained with the 1st perfusion. As demonstrated in the left area of Fig. 3-B, when the 2nd nicotine solution was perfused 5 min after washing out the drugfree solution with $286 \mathrm{mM}$ and $86 \mathrm{mM}$ sodium solution, being made equiosmotic by addition of glucose $(120 \mathrm{mM})$, the output of noradrenaline was $84.9 \pm 13.1$ and $92.6 \pm 16.3 \%$ of that released with the 1 st procedure, respectively.

When the 2nd nicotine solution was perfused into the solution containing guanethidine $2 \times 10^{-5} 30 \mathrm{~min}$ after the perfusion, the output of noradrenaline was markedly reduced 
$(3.6 \%, \mathrm{n}-2)$. When the 2 nd nicotine solution was perfused to normal sodium solution $5 \mathrm{~min}$ after washing out the guanethidine solution, however, the output of noradrenaline was reduced to $35.5+1.7 \%$ of that released with the 1 st procedure (lined column). This value was regarded as control of the drug action, which remained after the drug had been washed out. The action of guanethidine was significantly $(\mathrm{P}<0.01)$ accentuated by washing with $286 \mathrm{mM}$ sodium solution $\left(8.8 \div 1.3_{0}^{\circ}\right)$, as well as being moderately accentuated with $215 \mathrm{mM}$ sodium solution (23.8\%). On the other hand, it was significantly reduced $(\mathrm{P}<0.01)$ by washing with $86 \mathrm{mM}$ sodium solution $\left(66.0_{-}-4.0 \%\right)$.

Hearts were washed with $86 \mathrm{mM}$ sodium solution for $5 \mathrm{~min}$, and then perfused with normal sodium solution as demonstrated in the extreme right column of Fig. 3-B. When the 2 nd nicotine solution was perfused $5 \mathrm{~min}$ after, the output of noradrenaline was 29.4 ; $2.6 \%$ of that released with the 1 st nicotine perfusion. This value did not differ from control regarding the action of guanethidine.

Effect of washing with low or high potassium solution on guanethidine-induced decrease in output of noradrenaline released by nicotime in the isolated heart of rabbits

When the 2 nd nicoline solution was perfused into a low potassium solution ( $3.5 \mathrm{mM}$ ) 5 min after washing out the drug-free solution (potassium $4.7 \mathrm{mM}$ ), the output of noradrenaline was $61.1: 5.0 \%(n-4)$ of that released with the 1 st nicotine perfusion, this difference being significant $(\mathrm{P}<0.05)$ from that in untreated hearts $(95.3-14.1 \%$, the extreme left column in Fig. 3-B). The low polassium could cause hyperpolarization of the nerve membrane and antagonize the sustained depolarization induced by nicotine (13). When the 2nd nicotine solution was added to the low potassium solution 5 min after washing out the guanethidine solution $\left(2 \times 10^{5}\right)$, the output of noradrenaline was reduced to $25.1+2.7 \%(n-4)$ of that released with the 1 st nicotine perfusion, the difference being also significant $(P<0.05)$ from the control regarding the action of the drug $\left(35.5 \quad 1.7^{\circ} \%\right.$ lined column in Fig. 3-B).

On the other hand, when the 2 nd nicotine solution was perfused while the hearts were being perfused with a high potassium solution $(23.4 \mathrm{mM}) 5$ min after washing out the drug solution, the output of noradrenaline was $36.6 \pm 2.5 \%(\mathrm{n}-3)$ of that releused with the 1 st nicotine perfusion, the difference thus being insignificant.

\section{DISCUSSION}

The adrenergic neuron blocking action of guanethidine was reduced when the calcium level was raised simultaneously with the drug addition. The result is consistent with the findings reported by other investigators $(4,5,14)$. When nerve stimulation is done in the presence of high calcium, however, a simple interaction between calcium and guanethidine should be taken into account, as high calcium increases noradrenaline released from the sympathetic nerve $(15,16)$ and the drug reduces noradrenaline $(2,7,17)$. The present result may be attributed to a physiological antagonism between calcium and guanethidine on the output of the transmitter. In order to clarify the essential interaction other 
than the antagonism, nerve stimulation should be done in the presence of normal calcium after washing out high calcium $(2,5)$. Atrial responses to sympathetic nerve stimulation, which had been reduced by the pretreatment with guanethidine $\left(4 \times 10^{-5} \mathrm{M}\right)$, were not reversed when the drug was washed out and the concentration of calcium was raised to $50 \mathrm{mM}$ for $30 \mathrm{~min}$, after which the calcium concentration returned to normal. The irreversibility of the action of the drug was not due to a marked inhibition of the high calcium itself on heart rate, which appears to be a membrane stabilizing action (18). The result is not consistent with the findings reported by Kirpekar et al. (2) that the guanethidine $\left(1.2 \times 10^{-6}\right)$-induced abolition of noradrenaline released by stimulation of the splenic nerve of cat was restored to approx. $50 \%$ of control when the drug was washed out and calcium concentration was raised to $50 \mathrm{mM}$ for 20 to $30 \mathrm{~min}$, after which the high calcium concentration returned to normal. This inconsistency may be derived from differences in doses of the drug, parameters of experiments, organs or species. Various findings have been reported by different investigators in reference to interactions between the action of guanethidine and calcium $(2-5,14)$. No essential interaction between the action of guanethidine $4 \times 10^{-5}$ and $50 \mathrm{mM}$ calcium was found in the parameter of atrial positive chronotropic responses to nerve stimulation in rabbits.

Boura and Green (6) attributed the mode of action of adrenergic neuron blockers to a conduction blockade in the nerve terminals. The hypothesis was supported by Haeusler et al. $(19,20)$. The present results, however, show that the situation is indecd more complex. Hodgkin and Katz (21) demonstrated that in squid giant axon, the reversed potential difference of the active membrane depended on the external sodium concentration, that the conduction velocity underwent a decrease in low sodium solution and that the height of the action potentials was increased by a solution containing additional sodium chloride. Thus, if the conduction blockade induced by guanethidine is a major factor for its adrenergic neuron blockade, then the blocking action of the drug would be accentuated by lowering sodium and reduced by raising the ion. In fact, the adrenergic blockade induced by guanethidine was accelerated by perfusion with the solution containing low sodium $(86 \mathrm{mM})$ together with the drug addition, within the time when the low sodium itself produced no decrease in responses to nerve stimulation. These results partially support the above-mentioned hypothesis $(6,19,20)$. It should be noted, however, that nerve stimulation was carried out while perfusion with the solution containing guanethidine was being given. On the other hand, the action of the drug was not delayed or reduced by simultaneous perfusion with the high sodium $(286 \mathrm{mM})$, the same of which can not be attributed to hypertonicity of the solution. It was confirmed that in the present preparations, a complete adrenergic blockade was induced by tetrodotoxin $\left(6.2 \times 10^{-8}\right)$ 15 min after the application, and was significantly $(\mathbf{P}<0.05)$ delayed by the simultaneous perfusion with the high sodium (8). There is the case that acute partial sympathetic blockade by reserpine was reduced by perfusion with high sodium (9). These results provided the speculation that guanethidine itself may have an action against adrencrgic blockade due to its local anesthetic action, and the fact of no reduction of the guanethidine action 
induced by the high sodium could be attributed to being in the balance between the dual actions of the drug. The dissociation of the primary action of guanethidine from its local anesthetic action is reported to have been observed in the sympathetically innervated organs and the sensory $\mathrm{C}$ fibers $(22,23)$.

On the other hand, Haeusler et al. (20) have demonstrated in the heart of cats, that when guanethidine blocked the firing normally induced by a lower concentration of acetylcholine, antidromic discharges could still be obtained by a higher concentration of acetylcholine, and concluded that the adrenergic neuron blocking action of guanethidine may well be explained by the combination of its local anesthetic action and its accumulation in nerve terminals. They applied acetylcholine while perfusing with guanethidine. In the present experiments, high concentrations of $\mathrm{KCl}$ were applied to induce depolarization of the nerve membrane after guanethidine in extra- or intracellular space to be easily washed away had been washed out. The action of guanethidine was not reversible but reduced progressively after washing out, which differed from that of high doses of cocaine and procaine. As reported previously (9), reserpine $\left(1.6 \times 10^{-6}\right)$ markedly reduced noradrenaline released by $100 \mathrm{mM} \mathrm{KCl}$, but not reduction was seen with $300 \mathrm{mM} \mathrm{KCl}$ in the same experimental design. Different from the action of reserpine, however, guanethidine did not produce a 'ceiling effect' of the decrease in noradrenaline released by the higher concentration of $300 \mathrm{mM} \mathrm{KCl}$. It is feasible that the action of guanethidine which remained after the drug had been washed out is not due to an antagonistic action against depolarization of the nerve membrane induced by $\mathrm{KCl}$, i.e. it is not due to a membrane stabilizing action. This experimental design can be applied to further investigations in order to clarify the action of guanethidine itself against its local anesthetic action.

Guanethidine-induced decreases in positive chronotropic responses to electrical stimuli and noradrenaline released by nicotine were reversed by subsequent perfusion with the solution containing low sodium $(86 \mathrm{mM})$. There is much evidence supporting the fact that sodium is required for the uptake and storage of noradrenaline by the sympathetic nerve endings (24-27). One possible explanation for the results presented herein is that a decrease in the re-uptake of noradrenaline induced by the perfusion with the low sodium resulted in an increase in the recovery of the transmitter. This is hardly feasible, however, since the output of noradrenaline was not potentiated by the perfusion with the low sodium solution alone.

There is the possibility that restoration from the action of guanethidine can be attributed to an easier removal of the drug from a site of action by an unknown mechanism in a specific condition of the low sodium, for example, as sodium deficient media produce an increase in efflux of radioactive noradrenaline from prelabelled heart slices (25-27). The result herein (the extreme right column in Fig. 3-B), however, shows that such is not the case. The inhibitory action of guanethidine on the output of noradrenaline reappeared when nicotine was added to normal sodium solution after hearts had been perfused with the drug solution, and then with the drug-free low sodium solution. A decreased amount of guanethidine would hardly result in the reappearance of its action. 
Guanethidine is taken up into the sympathetic nerve endings by the same uptake process for noradrenaline (28-30). Gulati and Jaykar (31) reported the possibility that in the isolated rabbit's ileum, prevention from adrenergic neuron blockade could be due to the decrease in the uptake of guanethidine itself caused by previous or simultaneous incubation with a sodium free medium. This does not, however, explain the restoration from the action of guanethidine in the present experiment, induced by the subsequent perfusion with the low sodium solution.

Guanethidine-induced decreases in responses to electrical stimuli and noradrenaline released by nicotine were accentuated by the subsequent perfusion with the high sodium solutions. The potentiating effect of the high sodium was not due to hypertonicity of the solution but rather to the action of sodium itself. Lithium was not effective in replacing the action of sodium.

From an above-mentioned relationship between the concentrations of sodium and the action of guanethidine, a probable explanation is that guanethidine increases the permeability of the membrane of the endings to sodium ions, thereby leading to adrenergic neuron blockade. Such potency of guanethidine was demonstrated in the membrane of the isolated skin of frogs (32) and of atria isolated from rabbits pretreated with reserpine (33). Misu (33) demonstrated a biphasic action of guanethidine $4 \times 10^{-4}$ on the transmembrane potential of left driven atria. The maximum rate of rise of action potential significantly increased without hyperpolarization up to $30 \mathrm{~min}$ after its application, and then the resting potential gradually decreased. The former action of guanethidine was blocked by the pretreatment with $1.6 \times 10^{-7}$ and the latter action was inhibited by the addition of 3.1 and $6.2 \times 10^{-6}$ of tetrodotoxin.

Keen and Bogdanski (27) have suggested an inhibitory and antagonistic action of sodium to calcium on a postulated receptor site in the release of noradrenaline. Since there is presumably no essential interaction between the action of guanethidine and calcium, it would be feasible that guanethidine increases the permeability of the membrane of the adrenergic nerve terminals to sodium ions and increases the concentration or availability of sodium to compete with calcium at some site in the process by which the transmitter is released. Perfusion with a low potassium solution did not modify the action of guanethidine.

It is noteworthy that the drug is particularly of therapeutic value in the treatment of hypertensive patients, where the sodium level in extracellular fluid is highly elevated.

Acknowledgment: The authors are grateful to Prof. H. Takagi for his helpful advice and criticism.

\section{REFERENCES}

1) Maxwell, R.A., Plummer, A.J., Schneider, F., Povalski, H. and Daniel, A.I.: J. Pharmacol. exp. Ther. 128, 22 (1960)

2) Kirpekar, S.M., Wakade, A.R., Dixon, W. and Prat, J.C.: J. Pharmacol. exp. Ther. 165, $166(1969)$

3) Bouluiv, D.J.: Br. J. Pharmacol. Chemother. 32, 145 (1968) 
4) Wilson, J.: J. Pharm. Pharmacol. 22, 561 (1970)

5) Chang, C.C., Lat, F.M. and Chiuen, C.C.: Arch. int. Pharmacodyn. Thér. 190, 34 (1971)

6) Boura, A.L.A. and Green, A.F.: Ann. Rev. Pharmacol. 5, 183 (1965)

7) Kubo, T, AND Misu, Y.: Folia pharmacol, japon. 67, 40 p (1971) (in Japanese)

8) Misu, Y. ANd Nishio, H.: Folia pharmacol. japon. 67, 207 p (1971) (in Japanese)

9) Misu, Y., Kubo, T. and Nishio, H.: Eur. J. Pharmacol. 19, 267 (1972)

10) Misu, Y. and KirpeKar, S.M.: J. Pharmacol. exp. Ther. 163, 330 (1968)

11) Garry, R.C. and Gillespie, J.S.: J. Physiol. 128, 557 (1955)

12) Anton, A.H. And Sayre, D.F.: J. Pharmacol. exp. Ther. 138, 360 (1962)

13) Hafusler, G., Thofnfan, H., Haffely, W. nnd Hlerlimann, A.: Arch. exp. Path. Pharmak. 261, 389 (1968)

14) Burn, J.H. And Welsh, F.: Br. J. Pharmacol. Chemother. 31, 74 (1967)

15) KirpekAr, S.M. AND Misu, Y.: J. Physiol. 188, 219 (1967)

16) Misu, Y. And Kubo, T.: Japan. J. Pharmacol. 22, 359 (1972)

17) Huković, S. and Muschold, E.: Arch. exp. Path. Pharmak. 244, 81 (1962)

18) Shanes, A.M.: Pharmacol. Rev. 10, 165 (1958)

19) Hatusllir, G., Haffely, W. and Huerlimann, A.: Arch. exp. Path. Pharmak. 265, 260 (1969)

20) Haeusler, G., Thoenen, H. Hajeely, W. and Huerlimann, A.: Helv. physiol pharmacol. Acta 26, CR 352 (1968)

21) Hodgikt, A.L. And Katz, B.: J. Physiol. 108, 37 (1949)

22) Rand, M.J. and Wilson, J.: Eur. J. Pharmacol. 1, 200 (1967)

23) Watson, P.J.: Eur. J. Pharmacol. 1, 407 (1967)

24) Iversen, L.L. and Kravitz, E.A.: Mol. Pharmacol. 2, 360 (1966)

25) Gillis, C.N. and Paton, D.M.: Br. J. Pharmacol. Chemother. 29, 309 (1967)

26) Bocidanski, D.F. ANd Brodif, B.B.: J. Pharmacol. exp. Ther. 165, 181 (1969)

27) Keen, P.M. and Bogdanski, D.F.: Am. J. Physiol. 219, 677 (1970)

28) Chang, C.C., Costa, E. and Brodif, B.B.: J. Pharmacol. exp. Ther. 147, 303 (1965)

29) Obianwu, H.O., Stttzel, R. and Lundborg, P.: J. Pharm. Pharmacol. 20, 585 (1968)

30) Maítre, L. anid Stafhelin, M.: Biochem. Pharmacol. 22, 1233 (1971)

31) Gulati, O.D. And JaYKar, S.: Br. J. Pharmacol. Chemother. 42, 352 (1971)

32) Baba, W.I. and Suith, A.J.: Q. Il. exp. Physiol. Cog. Med. Sci. 49, 194 (1964)

33) Misu, Y.: Japan. J. Pharmacol. 24, Suppl. 132 (1974) 